International Mathematical Forum, 1, 2006, no. 30, 1491 - 1496

\title{
Nonnegative solutions for a class of Neumann-Robin BV semipositone problems
}

\author{
G. A. Afrouzi \\ Dept. of Math., Faculty of Basic Sciences, \\ Mazandaran University, Babolsar, Iran \\ e-mail: afrouzi@umz.ac.ir \\ M. Khaleghy Moghaddam \\ Dept. of Math., Faculty of Basic Sciences, \\ Mazandaran University, Babolsar, Iran \\ e-mail: m.moghadam@umz.ac.ir
}

\begin{abstract}
In this paper we study the following Neumann-Robin boundary value problem

$$
\left\{\begin{array}{l}
-u^{\prime \prime}(x)=\lambda f(u(x)), \quad x \in(0,1) \\
u^{\prime}(0)=0 \\
u^{\prime}(1)+\alpha u(1)=0,
\end{array}\right.
$$

where $\alpha<0, \lambda>0$ are parameters and $f \in C^{2}[0, \infty)$ such that $f(0)<$ 0 , and also $f$ is increasing and concave up. By using the quadrature method, we shall investigate the existence and multiplicity of results and in particular, we shall search how the difference of results behave with respect to different $\alpha^{6} s$.
\end{abstract}

Mathematics Subject Classification: 34B15

Keywords: semipositone problems, nonnegative solutions, quadrature method.

\section{Introduction}

We study the nonlinear two point boundary value problem

$$
-u^{\prime \prime}(x)=\lambda f(u(x)), \quad x \in(0,1)
$$




$$
\begin{aligned}
& u^{\prime}(0)=0, \\
& u^{\prime}(1)+\alpha u(1)=0,
\end{aligned}
$$

where $\alpha<0, \lambda>0$ are parameters, $f$ is a twice differentiable function on $[0, \infty)$ and $f(0)<0$ (i.e. semipositone problem), and we shall assume that $f^{\prime}(s) \geq$ $0, f^{\prime \prime}(s)>0, F(s)=\int_{0}^{s} f(t) d t$. So there exist unique values of $\beta, \theta>0$ such that $f(s)<0$ on $[0, \beta), f(\beta)=0$ and $F(\theta)=0$. The existence and multiplicity results is studied in [2] and [5] for cases $\alpha>0$ and $\alpha=0$ respectively. Also other types of two point boundary value problems have been studied in [1], [3], [4]. We use the quadrature method and establish the existence of nonnegative solutions $u(x)$ for the cases $u(x)=0$ and $u(x)=\theta$ in sections 2 .

\section{Main Results}

In this section we shall characterize our nonnegative solutions by the value of the solution at $x=0$, and denote it by $p$, i.e. $u(0)=p$. We show that for given $n=0,1,2, \ldots$ and $p=0, p=\theta$ (1)-(3) have two nonnegative solutions. One has even number i.c.p.'s (i.e. interior critical points) and the other has odd number i.c.p's as described in the following theorem.

Theorem 2.1. Let $f(0)<0, \alpha<0$, and $n=0,1,2, \ldots$ and $p=0(p=\theta)$ then there exists a real number $m^{*}$ such that:

The equations (1)-(3) have exactly two nonnegative solutions, one is $u_{1}$ with $k=2 n(k=2 n+1)$ i.c.p. 's at $\lambda_{1}=\lambda\left(p, m^{*}\right)$, where $\lambda_{1}=\lambda\left(p, m^{*}\right)$, is

$$
\sqrt{\lambda_{1}}=\frac{k}{\sqrt{2}} \int_{0}^{\theta} \frac{1}{\sqrt{-F(s)}} d s+\frac{1}{\sqrt{2}} \int_{0}^{-\frac{m^{*}}{\alpha}} \frac{1}{\sqrt{-F(s)}} d s \text { and } \sqrt{\lambda_{1}}=\frac{m^{*}}{\sqrt{2} \sqrt{-F\left(-\frac{m^{*}}{\alpha}\right)}}
$$

and the corresponding solution is defined by

$$
\int_{u_{1}(0)}^{u_{1}(x)} \frac{1}{\sqrt{-F(s)}} d s=\kappa \sqrt{2 \lambda_{1}} x ; \quad x \in\left(0, x_{0}\right) \quad \kappa=+(\kappa=-)
$$

and

$$
\int_{u_{1}(x)}^{u_{1}(1)} \frac{1}{\sqrt{-F(s)}} d s=\sqrt{2 \lambda_{1}}(1-x) ; \quad x \in\left(k x_{0}, 1\right)
$$

where $x_{0}=x_{0}\left(p, m^{*}\right)$, is

$$
x_{0}=\frac{\int_{0}^{\theta} \frac{1}{\sqrt{-F(s)}} d s}{k \int_{0}^{\theta} \frac{1}{\sqrt{-F(s)}} d s+\int_{0}^{-\frac{m^{*}}{\alpha}} \frac{1}{\sqrt{-F(s)}} d s}
$$


and $u_{1}^{\prime}(1)=m^{*}$ and the other nonnegative solution is $u_{2}$ with $k=2 n+1$ $(k=2 n)$ i.c.p.'s at $\lambda_{2}=\lambda(p, 0)$ where $\lambda_{2}=\lambda(p, 0)$ is

$$
\sqrt{\lambda_{2}}=\frac{k+1}{\sqrt{2}} \int_{0}^{\theta} \frac{1}{\sqrt{-F(s)}} d s
$$

and the corresponding solution is defined by

$$
\int_{u_{2}(0)}^{u_{2}(x)} \frac{1}{\sqrt{-F(s)}} d s=\kappa \sqrt{2 \lambda_{2}} x ; \quad x \in\left(0, x_{1}\right) \quad \kappa=+(\kappa=-)
$$

where $x_{1}=\frac{1}{k+1}$ and $u_{2}^{\prime}(1)=0$. Further note that $\lambda_{1}<\lambda_{2}\left(\lambda_{1}>\lambda_{2}\right)$ and $x_{1}<x_{0}\left(x_{1}>x_{0}\right)$.

Proof. Let $u(x)$ be a nonnegative solution of (1)-(3) with $u(0)=0(u(0)=\theta)$. Note that if $u(1)=0, u^{\prime}(1)=0$ and if $u(1) \neq 0$, then $u^{\prime}(1)>0$. Also $u(x)$ must be initially increasing and concave up (down), and be finally increasing if $u^{\prime}(1)>0$ and be finally decreasing if $u^{\prime}(1)=0$. Hence $u(x)$ can have either an even number of i.c.p.'s or an odd number of i.c.p.'s. Since (1) is autonomous, every solution of (1)-(3) is symmetric about each of its i.c.p.'s. Hence we study only solution $u_{1}$, in $\left[0, x_{0}\right]$ and $\left[k x_{0}, 1\right]$ where $k=2 n(k=2 n+1)$ and $x_{0}$ is the first i.c.p.

We shall first discuss the case where $u^{\prime}(1)>0$ thus $u(1)=-\frac{m}{\alpha} \in(0, \theta)$. Suppose $u(0)=u\left(2 x_{0}\right)=\cdots=p$. Thus $u\left(x_{0}\right)=u\left(3 x_{0}\right)=\cdots=q$ where $q=\theta(q=0)$ if $p=0(p=\theta)$. Now multiplying both sides of (1) by $u^{\prime}(x)$ and integrating, we obtain

$$
-\frac{\left[u^{\prime}(x)\right]^{2}}{2}=\lambda F(u(x))+c .
$$

Since $u(0)=p, c=-\lambda F(u(0))=0$, so we have

$$
-\frac{\left[u^{\prime}(x)\right]^{2}}{2}=\lambda[F(u(x)] ; \quad x \in(0,1)
$$

and therefore

$$
u^{\prime}(x)=\kappa \sqrt{2 \lambda} \sqrt{-F(u(x))} ; \quad x \in\left(0, x_{0}\right) \quad \kappa=+(\kappa=-)
$$

and

$$
u^{\prime}(x)=\sqrt{2 \lambda} \sqrt{-F(u(x))} ; \quad x \in\left(k x_{0}, 1\right) .
$$

Now integrating $(4)$ and $(5)$ on $\left(0, x_{0}\right)$ and $\left(k x_{0}, 1\right)$, respectively, we obtain

$$
\int_{u(0)}^{u(x)} \frac{1}{\sqrt{-F(s)}} d s=\kappa \sqrt{2 \lambda} x ; \quad x \in\left(0, x_{0}\right) \quad \kappa=+(\kappa=-)
$$




$$
\int_{u(x)}^{u(1)} \frac{1}{\sqrt{-F(s)}} d s=\sqrt{2 \lambda}(1-x) ; \quad x \in\left(k x_{0}, 1\right)
$$

Since $u^{\prime}(1)>0$, let $u^{\prime}(1)=m$ where $m>0$. Thus $u(1)=-\frac{m}{\alpha} \in(0, \theta)$. Substituting $x=x_{0}$ and $x=k x_{0}$ in (6) and (7), respectively, and multiplying (6) by $k$ and adding it to (7), we see

$$
\sqrt{\lambda}=\frac{k}{\sqrt{2}} \int_{0}^{\theta} \frac{1}{\sqrt{-F(s)}} d s+\frac{1}{\sqrt{2}} \int_{0}^{-\frac{m}{\alpha}} \frac{1}{\sqrt{-F(s)}} d s=\frac{A(m)}{\sqrt{2}} .
$$

Combining (6) and (8) we get

$$
x_{0}=\frac{\int_{0}^{\theta} \frac{1}{\sqrt{-F(s)}} d s}{k \int_{0}^{\theta} \frac{1}{\sqrt{-F(s)}} d s+\int_{0}^{-\frac{m}{\alpha}} \frac{1}{\sqrt{-F(s)}} d s} .
$$

Also substituting $x=1$ in (5), we have

$$
\sqrt{\lambda}=\frac{m}{\sqrt{2} \sqrt{-F\left(-\frac{m}{\alpha}\right)}}=\frac{B(m)}{\sqrt{2}} .
$$

Combining (8) and (10), for such a solution to exist, there must exist an $m^{*}$ such that $A\left(m^{*}\right)=B\left(m^{*}\right)$ Now we investigate whether such $m^{*}$ exists. Now define $L(m)=B(m)-A(m)$ on $(0,-\alpha \theta)$, i.e.

$$
L(m)=\frac{m}{\sqrt{-F\left(-\frac{m}{\alpha}\right)}}-k \int_{0}^{\theta} \frac{1}{\sqrt{-F(s)}} d s-\frac{1}{\sqrt{2}} \int_{0}^{-\frac{m}{\alpha}} \frac{1}{\sqrt{-F(s)}} d s .
$$

Then $L(m) \rightarrow \infty$ as $m \rightarrow-\alpha \theta^{-}$and $L(m) \rightarrow-k \int_{0}^{\theta} \frac{1}{\sqrt{-F(s)}} d s$ as $m \rightarrow 0^{+}$and

$$
L^{\prime}(m)=\frac{2(\alpha+1)[-F(-m / \alpha)]-m f(-m / \alpha)}{2 \alpha[-F(-m / \alpha)]^{3 / 2}} .
$$

For $m \in(0,-\alpha \theta)$ define $K(m)=2(\alpha+1)[-F(-m / \alpha)]-m f(-m / \alpha)$. Then $K^{\prime}(m)=\frac{\alpha+2}{\alpha} f(-m / \alpha)+m / \alpha f^{\prime}(-m / \alpha)$.

If $-1<\alpha<0$ it is easy to see that $L^{\prime}(m)<0$ on $(0,-\alpha \beta]$. Also $K^{\prime}(-\alpha \beta)=$ $-\beta f^{\prime}(\beta)<0$ and $K^{\prime \prime}(m)<0$ on $(-\alpha \beta,-\alpha \theta)$. Then $K^{\prime}(m)<0$ on $(-\alpha \beta,-\alpha \theta)$ and since $K(-\alpha \beta)>0$ and $K(-\alpha \theta)=\alpha \theta f(\theta)<0$, then there exists a unique $c \in(-\alpha \beta,-\alpha \theta)$, such that $L^{\prime}(m)<0$ on $(-\alpha \beta, c), L^{\prime}(m)>0$ on $(c,-\alpha \theta)$ and $L^{\prime}(c)=0$. If $\alpha=-1$, it is easy to see that $L^{\prime}(m)<0$ on $(0, \beta), L^{\prime}(\beta)=0$ and $L^{\prime}(m)>0$ on $(\beta, \theta)$. Note that in this case, $K(m)=-m f(m)$ and $K(c)=0$ at $c=\beta$. If $-2<\alpha<-1$, it is easy to see that $L^{\prime}(m)>0$ on $[-\alpha \beta,-\alpha \theta)$. Also $K^{\prime \prime}(m)<0$ on $(0,-\alpha \beta)$ and $K^{\prime}(-\alpha \beta)=-\beta f^{\prime}(\beta)<0$ and 
$K^{\prime}(0)=\frac{\alpha+2}{\alpha} f(0)>0$. Since $f(0)<0$ and $\frac{\alpha+2}{\alpha}<0$. Hence $K^{\prime}(m)<0$ on $(0,-\alpha \beta)$. On the other hand $K(0)=0$ and $K(-\alpha \beta)=2(\alpha+1)[-F(\beta)]<0$, hence there exists a unique $c \in(0,-\alpha \beta)$ such that $L^{\prime}(m)<0$ on $(0, c)$ and $L^{\prime}(c)=0$ and $L^{\prime}(m)>0$ on $(c,-\alpha \beta)$. If $\alpha \leq-2$, then $L^{\prime}(m)>0$ on $m \in(-\alpha \beta,-\alpha \theta)$. Also $K^{\prime \prime}(m)<0$ on $(0,-\alpha \beta)$ and $K^{\prime}(0)=\frac{\alpha+2}{\alpha} f(0)<0$. Thus $K^{\prime}(m)<0$ on $(0,-\alpha \beta)$. Then $K(m)<0$ on $(0,-\alpha \beta)$, since $K(0)=0$. Therefore $L^{\prime}(m)>0$ on $(0,-\alpha \beta)$. Thus for any $\alpha<0$, there exists a unique $m^{*}=m^{*}(\alpha, \theta) \in(0,-\alpha \theta)$ such that $L\left(m^{*}\right)=0$ and hence $A\left(m^{*}\right)=B\left(m^{*}\right)$. Therefore we obtain a unique $\lambda_{1}$ where $\lambda_{1}=\left[A\left(m^{*}\right)\right]^{2}$ such that one can prove that (1)-(3) have the solution $u_{1}$ with $k$ i.c.p.'s at $\lambda=\lambda_{1}$, defined by (6), (7) and (9) with $u_{1}(0)=p, u_{1}^{\prime}(1)=m^{*}$ at $\lambda_{1}$ given by (8) or (10).

Now we study a solution satisfying $u(0)=0(u(0)=\theta)$ and $u(1)=u^{\prime}(1)=0$ with $k=2 n+1(k=2 n)$ i.c.p.'s. Hence $x_{1}=\frac{1}{k+1}$ and the problem is equivalent to studying a nonnegative solution of problem $-u^{\prime \prime}(x)=\lambda f(u(x)) ; \quad x \in\left(0, x_{1}\right)$ with boundary value conditions $u(0)=0=u^{\prime}\left(x_{1}\right)\left(u\left(x_{1}\right)=0=u^{\prime}(0)\right)$.

Now we again apply the quadrature technique and it easily follows that (1)-(3) have a solution, defined by

$$
\int_{u(0)}^{u(x)} \frac{1}{\sqrt{-F(s)}} d s=\kappa \sqrt{2 \lambda} x ; \quad x \in\left(0, x_{1}\right) \quad \kappa=+(\kappa=-)
$$

at $\lambda_{2}=\lambda(p, 0)$ where $\lambda_{2}=\lambda(p, 0)$ is

$$
\sqrt{\lambda_{2}}=\frac{k+1)}{\sqrt{2}} \int_{0}^{\theta} \frac{1}{\sqrt{-F(s)}} d s
$$

We know that

$$
\sqrt{\lambda_{1}}=\frac{k}{\sqrt{2}} \int_{0}^{\theta} \frac{1}{\sqrt{-F(s)}} d s+\frac{1}{\sqrt{2}} \int_{0}^{-\frac{m^{*}}{\alpha}} \frac{1}{\sqrt{-F(s)}} d s
$$

and $0<-\frac{m}{\alpha}<\theta$ and $\frac{1}{\sqrt{-F(s)}}>0$. Hence

$$
\frac{1}{\sqrt{2}} \int_{0}^{\theta} \frac{1}{\sqrt{-F(s)}} d s>\frac{1}{\sqrt{2}} \int_{0}^{-\frac{m^{*}}{\alpha}} \frac{1}{\sqrt{-F(s)}} d s
$$

and it easily follows from (12) and (13) that $\lambda_{1}<\lambda_{2}\left(\lambda_{1}>\lambda_{2}\right)$. We know $k x_{0}+l=1=(k+2) x_{1},\left(\right.$ or $\left.k x_{0}+l=1=k x_{1}\right)$ where $k=2 n(k=2 n+1)$ and $x_{0}>l>0$. Thus $x_{0}>x_{1}\left(x_{0}<x_{1}\right) . \triangle$ 


\section{References}

[1] V. Anuradha, C. Brown and R. Shivaji, Explosive nonnegative solutions to two point boundary value problems, Nonlinear Anal. 26, No. 3 (1996), 613-630.

[2] V. Anuradha, C. Maya and R. Shivaji, Positive solutions for a class of nonlinear boundary value problems with Neumann-Robin boundary conditions, J. Math. Anal. Appl. 236 (1999), 94-128.

[3] V. Anuradha and R. Shivaji, Sign changing solutions for a class of superlinear multiparameter semipositone problems, Nonlinear Anal. 24, No. 11 (1995), 1581-1596.

[4] A. Castro and R. Shivaji, Nonnegative solutions for a class of non-positone problems, Proc. Roy. Soc. Edinburgh, Sect. A 108 (1988),291-302.

[5] A. R. Miciano and R. Shivaji, Multiple Positive solutions for a class of semipositone Neumann two point boundary value problems, J. Math. Anal. Appl. 178 (1993), 102-115.

Received: Nov. 26, 2005 